\title{
The Reaction of Stock Markets to Crashes and Events: A Comparison Study between Emerging and Mature Markets using Wavelet Transforms.
}

\author{
Adel Sharkasi, Martin Crane, Heather J. Ruskin \\ School of Computing, Dublin City University, Dublin 9, Ireland \\ Email: asharkasi, mcrane and hruskin@computing.dcu.ie \\ Jose A. Matos \\ Faculdade de Economia, Universidade do Porto, 4200 Porto, Portugal \\ Email: jamatos@fep.up.pt
}

December 12, 2005

\begin{abstract}
We study here the behaviour of the first three eigenvalues $\left(\lambda_{1}, \lambda_{2}\right.$, $\left.\lambda_{3}\right)$ and their ratio $\left[\left(\lambda_{1} / \lambda_{2}\right),\left(\lambda_{1} / \lambda_{3}\right),\left(\lambda_{2} / \lambda_{3}\right)\right]$ of the covariance matrices of the original return series and of those rebuilt from wavelet components for emerging and mature markets. It has been known for some time that the largest eigenvalue $\left(\lambda_{1}\right)$ contains information on the risk associated with the particular assets of which the covariance matrix is comprised. Here, we wish to ascertain whether the subdominant eigenvalues $\left(\lambda_{2}, \lambda_{3}\right)$ hold information on the risk of the stock market and also to measure the recovery time for emerging and mature markets. To do this, we use the discrete wavelet transform which gives a clear picture of the movements in the return series by reconstructing them using each wavelet component. Our results appear to indicate that mature markets respond to crashes differently to emerging ones, in that emerging markets may take up to two months to recover while major markets take less than a month to do so. In addition, the results appears to show that the subdominant eigenvalues $\left(\lambda_{2}, \lambda_{3}\right)$ give additional information on market movement, especially for emerging markets and that a study of the behaviour of the other eigenvalues may provide insight on crash dynamics.
\end{abstract}


keywords: Variance-Covariance Matrix, Eigenvalues and Wavelet Analysis

\section{Introduction}

Covariance (Correlation) matrix forecasts of financial asset returns are an important component of current practice in financial risk management with a large bibliography on the subject. Meric and Meric (1997), for example, applied the Box M method and Principal Component Analysis (PCA) to test whether or not the correlation matrices before and after the international crash in 1987 were significantly different. This was done in order to investigate the changes in the long-term co-movements of twelve European and US equity markets. Their results showed that there are significant alterations in the co-movements of these markets and that the benefits of international diversification for the European markets decreased markedly after this crash.

Further, Kwapien et al. (2002) investigated the distribution of eigenvalues of correlation matrices for equally-separated time windows in order to study, quantitatively, the relation between stock price movements and properties of the distribution of the corresponding index motion (w.r.t. German DAX). They reported that the importance of an eigenvalue is related to the correlation strength of different stocks, which means that the more aggregated the market behaviour, the larger $\lambda_{1}$ (Maximum Eigenvalue).

Recently, in Keogh et al.(2003), we showed that periods in market sector data from the Dow Jones EURO-STOXX index, exist linearly with time. These results supported an implied relationship between volatility and the change in magnitude of the dominant eigenvalue and also showed that epochs seem to exist in all market sectors although in different degrees. More recently, Kwapien et al. (2004) analysed tick-by-tick returns data ranging from seconds up to 48 hours from the NYSE and the German markets. The authors compared the magnitude of the dominant eigenvalue of the correlation matrices for the same group of securities on various time scales. Their results indicated that collective market behaviour appears at significantly shorter time scales in recent times.

Pofka and Kondor (2002) examined the effect of noisy covariance matrices on the portfolio optimisation problem and found that the risk of the portfolio in the presence of noise in these matrices is $5-15 \%$ higher than in the absence of noise, indicating that the decrease in efficiency of the optimal portfolio is actually much less dramatic.

According to the findings of (Galluccio et al (1998), Laloux et al (1999), Plerou et al (1999), Laloux (2000), Plerou et al (2001), Wilcox and Gebbie (2004) and Sharifi et al (2004)), the correlation (or covariance) matrices 
of financial time series, apart from a few large eigenvalues and their corresponding eigenvectors, appear to contain such a large amount of noise that their structure can essentially be regarded as random. This means that a few of the larger eigenvalues might carry collective information. However, most previous studies (Gopikrishnan et al (2001))have focused on the largest eigenvalue with no attention paid to the others. If we are to presume that, as with any PCA analysis of data, there are several principal components that are significant, then it should be worth examining lesser order components to see if they can provide additional data for investment strategies. References in the literature to the role of higher order eigenmodes in investment strategy are scarce, but, recently Wilcox and Gebbie (2004) have examined the composition of all the eigenmodes of ten years of Johannesburg Stock Exchange using Random Matrix Theory (RMT). The authors concluded that "the leading [i.e. first three] eigenmodes may be interpreted in terms of independent trading strategies with long range correlations" indicating a role not just for $\lambda_{1}$ but also for a small number of the dominant eigenvalues. In the current work we aim, firstly, to highlight the apparent information obtained from the first two subdominant eigenvalues as well as the dominant eigenvalue and study its behaviour. Secondly, to compare the behaviour of the second and third eigenvalues for stock market indices for two different classes, namely emerging and mature markets.

Several studies have made comparisons between Emerging and Mature markets ${ }^{1}$, according to different characteristics, and these generally have reported that Emerging markets consistently behave differently to Mature ones. Patel and Sarkar (1998) studied eight mature and ten developing markets from 1970 to 1997. The authors found important differences in the characteristics of stock market crises between major and emerging markets. They also found that, for emerging markets, the decline in prices following crises is larger than that for mature markets, and the recovery time is longer. Further, Fuss (2002) used discriminant analysis to investigate if emerging and mature markets behave differently according to different financial aspects (such as market pricing, market size and market activity) and stated that the difference between these two market types has increased since the end of the 1990s. A reason for this could be found in financial crashes of 1994 in Mexico, 1997/1998 in Asia, 1998 in Russia and 1999 in Brazil, indicating that emerging and mature markets deal differently with crashes and crises.

\footnotetext{
${ }^{1}$ The International Finance Corporation (IFC) uses income per capita and market capitalization relative to GNP for classifying equity markets. If either (1) a market resides in a low- or middle-income economy, or (2) the ratio of investable market capitalization to GNP is low, then the IFC classifies the market as emerging otherwise IFC classifies it as mature.
} 
Recently, Salomons and Grootveld (2002) studied the equity risk premium in thirty-one global stock markets using standard statistical approaches and found that emerging markets carry a higher equity risk premium than mature ones indicating that they are perceived to be riskier. More recently, Wooldridge et al. (2003) considered the changes in the links between emerging and mature markets according to capital flows, the investor base and the changing character of global banks. Results showed that emerging and mature markets are more integrated nowadays than before. This contravenes with the findings of [Patel and Sarkar (1998), Fuss (2002), Salomons and Grootveld (2002) and Wooldridge et al. (2003)], so it is very important to carry out further investigations in order to clarify the issue.

In Sharkasi et al. (2004), we studied the behaviour of eigenvalues of the Covariance matrices around crashes and also studied the ratio of the dominant $\left(\lambda_{1}\right)$ to the subdominant $\left(\lambda_{2}\right)$ for emerging and mature markets. Our results showed that mature markets react to crashes in a different way than emerging ones which take longer to recover than mature markets. The second largest eigenvalue $\left(\lambda_{2}\right)$ may thus be expected to provide additional information on market movements.

Our objectives in this article are, therefore as follows:

1. To study the variation of the ratio series of the largest $\left(\lambda_{1}\right)$ to the second and third largest $\left(\lambda_{2}, \lambda_{3}\right)$ eigenvalues of the Variance-Covariance matrices for sliding windows of equal sizes for original return series of stock market indices. This, in order to compare the behaviour of this ratio across windows with different degrees of risk (or different crashes and events).

2. To study the previous point for return series which have been reconstructed using each wavelet component separately in order to measure how long the markets take to recover and how long these markets retain information about previous crises and events.

3. To study the variation of the largest and the second largest eigenvalues of these Covariance matrices for the original return series, and for those rebuilt from wavelet components, in order to see the direction of the movements in these markets and also to investigate whether $\lambda_{2}$ contains useful information about these movements, in addition to that described by $\lambda_{1}$ alone.

The remainder of this paper is organized as follows: The methodology used here is described briefly below (Section 2), with data and results presented in Section 3. The final section provides discussion and conclusion. 


\section{Methodology}

\subsection{How to Estimate Covariance Matrices}

There are several methods to compute the Variance-Covariance matrix but there is no agreement among authors on an optimal one. We have chosen the following formula because it uses weighted historical data to account for the empirical regularities of financial time series (such as the fact that volatility and correlation vary over time and these series have a "Fat Tail" distribution).

$$
\sigma_{i j}^{T}(M)=\left(\sum_{s=0}^{T} \omega_{T-s} r_{i, T-s} r_{j, T-s}\right) /\left(\sum_{s=0}^{T} \omega_{T-s}\right)
$$

where $r_{i, T}$ is the return on the $i^{\text {th }}$ market at date $T$ and $\omega_{T}$, which is the weight applied at date $T$ over horizon $M$, has been chosen to be a declining function of time. The more recent observations are given more weight than observations that occurred in more distant past, where $100 \%$ weight is given to the most recent week and each week in history receives $90 \%$ of the weight of the following week, then $\omega_{T}=1.0, \omega_{T-1}=0.90, \omega_{T-2}=0.81$, etc. (For more details see Litterman and Winkelmann (1998)).

In our study, we use weekly returns of a set of thirteen $(i=13)$ emerging indices and a set of fourteen $(i=14)$ mature indices and the VarianceCovariance matrices for overlapping windows of size $20(T=20)$ weeks for our data have been calculated using Equation(??) in order to study the structure change of stock market for different windows with different risk degree (i.e. after include the week of crash).

\subsection{Definition of Wavelet Transform}

The wavelet transform (WT) is a mathematical tool that can be applied to many applications such as image analysis, and signal processing. It was introduced to solve problems associated with the Fourier transform, as occur when dealing with non-stationary signals, or when dealing with signals which are localized in time or space as well as frequency. The wavelet transform has been explained in more detail, particularly in [Hijmans (1993), Bruce and Gao (1996) and Gonghui et al (1999) ].

In particular, the discrete wavelet transform (DWT) is useful in dividing the data series into components of different frequency, so that each component can be studied separately to investigate the data series in depth. The 
wavelets have two types, father wavelets $\phi$ and mother wavelets $\psi$ where

$$
\int \phi(t) d t=1 \text { and } \int \psi(t) d t=0
$$

The smooth and low-frequency parts of a signal are described by using the father wavelets, while the detail and high-frequency components are described by the mother wavelets. The orthogonal wavelet families have four different types which are typically applied in practical analysis, namely, the haar, daublets, symmlets and coiflets.

The following brief synopsis of their features is relevant to the analysis reported:

- The haar has compact support and is symmetric but, unlike the others, is not continuous.

- The daublets are continuous orthogonal wavelets with compact support.

- The symmlets have compact support and were built to be as nearly symmetric as possible.

- The coiflets were built to be nearly symmetric.

A two-scale dilation equation, used to calculate the wavelets, father $\phi(t)$ and mother $\psi(t)$, is defined respectively by

$$
\begin{aligned}
\phi(t) & =\sqrt{2} \sum_{k} \ell_{k} \phi(2 t-k) \\
\psi(t) & =\sqrt{2} \sum_{k} \hbar_{k} \phi(2 t-k)
\end{aligned}
$$

where $\ell_{k}$ and $\hbar_{k}$ are the low-pass and high-pass coefficients given by

$$
\begin{aligned}
\ell_{k} & =\frac{1}{\sqrt{2}} \int \phi(t) \phi(2 t-k) d t \\
\hbar_{k} & =\frac{1}{\sqrt{2}} \int \psi(t) \phi(2 t-k) d t
\end{aligned}
$$

The orthogonal wavelet series approximation to a signal $f(t)$ is defined by

$$
f(t)=\sum_{k} s_{J, k} \phi_{J, k}(t)+\sum_{k} d_{J, k} \psi_{J, k}(t)+\ldots+\sum_{k} d_{1, k} \psi_{1, k}(t)
$$

where $J$ is the number of multi-resolution levels (or scales) and $k$ ranges from 1 to the number of coefficients in the specified components (or crystals). The 
coefficient $s_{J, k}, d_{J, k}, \ldots, d_{1, k}$ are the wavelet transform coefficients given by

$$
\begin{gathered}
s_{J, k}=\int \phi_{J, k}(t) f(t) d t \\
d_{j, k}=\int \psi_{j, k}(t) f(t) d t \quad(j=1,2, \ldots, J)
\end{gathered}
$$

Their magnitudes give a measure of the contribution of the corresponding wavelet function to the signal. The functions $\phi_{J, k}(t)$ and $\psi_{j, k}(t)[j=$ $1,2, \ldots, J]$ are the approximating wavelet functions, generated from $\phi$ and $\psi$ through scaling and translation as follows

$$
\begin{gathered}
\phi_{J, k}(t)=2^{\frac{-J}{2}} \phi\left(2^{-J} t-k\right)=2^{\frac{-J}{2}} \phi\left[\left(t-2^{J} k\right) / 2^{J}\right] \\
\psi_{J, k}(t)=2^{\frac{-J}{2}} \psi\left(2^{-J} t-k\right)=2^{\frac{-J}{2}} \psi\left[\left(t-2^{J} k\right) / 2^{J}\right] \quad j=1,2, \ldots, J
\end{gathered}
$$

The discrete wavelet transform (DWT) is used to compute the coefficient of the wavelet series approximation in Equation(6) for a discrete signal $f_{1}, \ldots, f_{n}$ of finite extent. The DWT maps the vector $f=\left(f_{1}, f_{2}, \ldots, f_{n}\right)^{\prime}$ to a vector of $n$ wavelet coefficients $w=\left(w_{1}, w_{2}, \ldots, w_{n}\right)^{\prime}$ which contains both the smoothing coefficient $s_{J, k}$ and the detail coefficients $d_{j, k}[j=1,2, \ldots, J]$. The $s_{J, k}$ describe the underlying smooth behaviour of the signal at coarse scale $2^{J}$ while $d_{J, k}$ describe the coarse scale deviations from the smooth behaviour and $d_{J-1, k}, \ldots, d_{1, k}$ provide progressively finer scale deviations from the smooth behaviour.

In the case when $n$ is divisible by $2^{J}$; there are $n / 2$ observations in $d_{1, k}$ at the finest scale $2^{1}=2$ and $n / 4$ observations in $d_{2, k}$ at the second finest scale $2^{2}=4$. Likewise, there are $n / 2^{J}$ observations in each of $d_{J, k}$ and $s_{J, k}$ where

$$
n=n / 2+n / 4+\ldots+n / 2^{J-1}+n / 2^{J}+n / 2^{J} .
$$

We apply the discrete wavelet transform to split the weekly return series for emerging and mature market indices into different frequency components to get a clear picture of the movements in these markets. We also wish to rebuild the return series using the first three wavelet components $\left(d_{1}, d_{2}\right.$ and $d_{3}$ ) which explain more than $80 \%$ of energy (or magnitude) of these series $^{2}$ (see Tables 1 and 2) in order to study the fortnightly, monthly and bi-monthly data which are represented by $d_{1}, d_{2}$ and $d_{3}$ respectively.

\footnotetext{
${ }^{2}$ This means that $80 \%$ of the time series can be reconstructed by using $d_{1}, d_{2}$ and $d_{3}$
} 


\section{Data and Results}

\subsection{Data Description}

The data used in the following analysis consists of the weekly prices of a set of thirteen emerging market indices and a set of fourteen mature market indices during the period from the second week of January 1997 to the third week of March 2004. These markets are listed in Tables 1 and 2. As each market uses its local currency for presenting the index values, we use the weekly returns instead of using the weekly prices, where the following formula applies:

$$
\text { Weekly Return }=\operatorname{Ln}\left(P_{t} / P_{t-1}\right)
$$

and where

- $P_{t}$ is the closing price of the index at week $t$.

- $P_{t-1}$ is the closing price at week $t-1$.

\subsection{Empirical Results}

\subsubsection{Eigenanalysis for Original Return Series.}

The variation of the ratio of the Largest $\left(\lambda_{1}\right)$ to the Second Largest $\left(\lambda_{2}\right)$ eigenvalues of the Covariance matrices for equal overlapping time windows of the original returns series for emerging and mature markets, are shown in Figures 1(a) and 2(a) respectively. These show a qualitative difference in the way emerging and mature markets deal with crashes and events.

We also plot the ratio of $\lambda_{1}$ to $\lambda_{3}$ to see clearly the reactions of stock markets to different crashes and events. The variation of these ratios is plotted in Figures 3(a and b). It can be seen that the mature markets have reacted to events more strongly than emerging markets, especially after the $9 / 11$ crash, in order to regain stability and reduce risk to the markets. This means that mature markets effectively became anti-persistent, while emerging markets are persistent in agreement with the findings of Di Matteo et al (2003) and (2005) which indicate that emerging markets have $H \geq 0.5$, while mature markets have $H \leq 0.5$ [ $H$ is the Hurst exponent].

The ratios of $\lambda_{2}$ to $\lambda_{3}$, Figures 4 ( $\mathrm{a}$ and $\mathrm{b}$ ), are plotted in order to see if behavior for $\lambda_{2}$ and $\lambda_{3}$ differs for emerging and mature markets. In other words, we want to investigate whether or not $\lambda_{2}$ carries additional information about these different market types. Figures $4(\mathrm{a}$ and $\mathrm{b})$ suggest that ratios of $\lambda_{2}$ to $\lambda_{3}$ for emerging markets are more variable than those for 
mature markets, implying that subdominant $\left(\lambda_{2}\right)$, as well as dominant $\left(\lambda_{1}\right)$ eigenvalues, do play a part in describing the behaviour of emerging markets while the behaviour of mature markets is described by $\lambda_{1}$ only.

In comparing the ratio $\left(\lambda_{1} / \lambda_{2}\right)$, Figures $1(\mathrm{a})$ and $2(\mathrm{a})$, for emerging and mature markets, it can be seen that for latter, there are three highly significant points in the ratio variation which are for window numbers 120, 219 and 345 respectively. Window 120 starts from week 120 to week 139 which is the third week of October, 1999 (the $12^{\text {th }}$ anniversary of October 19, 1987 $\left.\operatorname{crash}^{3}\right)$. The last week in window 219 is week 238 which is the second week of September, 2001 (9/11 crash) and window 345 starts from week 345 to week 364 which is the third week of March, 2004 (Madrid Bomb). However, for emerging markets, there is only one highly significant point which is for window 212, where the last week in this window is the second week of September, 2001 (9/11 crash). We suggest that the cause for these highly significant ratio points is one or more of the following reasons:

1. Increasing the value of the largest eigenvalue $\left(\lambda_{1}\right)$ while the second largest eigenvalue $\left(\lambda_{2}\right)$ remains stable.

2. Decreasing the value of $\lambda_{2}$ while the value of $\lambda_{1}$ does not change.

3. Increasing the value of $\lambda_{1}$ while decreasing the value of $\lambda_{2}$, (or in other words, $\lambda_{1}$ and $\lambda_{2}$ moving in opposite directions).

The changes in $\lambda_{1}$ and $\lambda_{2}$ are plotted in Figure 5 (a and $\mathrm{b}$ ) for emerging and mature markets respectively. For mature markets, (in order to examine likely causes), we compared the values of $\lambda_{1}$ and $\lambda_{2}$ of the covariance matrix for windows 120, 219 and 345 with the values of the previous windows, while for emerging markets, we compared the values of $\lambda_{1}$ and $\lambda_{2}$ for window 212 with the values of the previous windows. We found that the third reason above causes peaks in emerging markets while it is the first driver for change in the mature markets. This implies that both $\lambda_{1}$ and $\lambda_{2}$ are important in describing the behaviour of emerging markets while $\lambda_{1}$ is sufficient alone to explain the behaviour of mature markets.

\footnotetext{
${ }^{3}$ This was the last October in $20^{t h}$ century and October is always hard month for stock markets so, with the end of the century as well, a crash in October was anticipated but did not happen. This, not least because, "The world markets were actually sent into turmoil by a speech by Alan Greenspan, and the Dow Jones for the first time since April 8, 1999 dipped below 10.000 on October 15 and 18, 1999. However, the market did not crash and instead quickly recovered and later started a renewed and strengthened bullish phase", Sornette (2002).
} 


\subsubsection{Eigenanalysis associated with Wavelet Transform.}

The discrete wavelet transform (DWT) with symmlet 8 wavelet $\left(s_{8}\right)$ for 6 levels (scales) is computed for weekly returns series of all indices for emerging and mature markets. The DWT provides a more detailed breakdown of the contribution to the series energy from the high and low frequencies in the following manner. Tables 1 and 2 display the energy (or magnitude) percentages explained by each wavelet component (crystal) of the original returns for emerging and mature market indices respectively. From Tables 1 and 2 , it can be seen that high-frequency crystals, especially the first three $\left(d_{1}, d_{2}\right.$ and $\left.d_{3}\right)$ have much more energy than the lowest frequency one $\left(s_{6}\right)$ implying that movements in these series are mainly caused by short-term fluctuations.

In order to measure the recovery time of emerging and mature markets from crashes and how long these markets retain information about crashes, we employed the discrete wavelet transform (DWT) and eigenanalysis. The steps of this process are: (i) Use the DWT to divide the return series of emerging and mature markets into different frequency components. (ii) Rebuild the returns using each wavelet components $\left(d_{1}, d_{2}, d_{3}\right.$, etc) and (iii) Study the distribution of the ratio $\left(\lambda_{1} / \lambda_{2}\right)$ of variance-covariance matrices for overlapping windows of size 20 for these series.

Figures 1 (b, c and d) and 2 (b, c and d) show the $\operatorname{ratio}\left(\lambda_{1} / \lambda_{2}\right)$ from covariance matrices for each window for the return series, which are rebuilt from $d_{1}, d_{2}$ and $d_{3}$, (representing fortnightly, monthly and bi-monthly data respectively), for emerging and mature markets respectively. Looking at the ratio scales in these Figures, we can clearly seen two main features; firstly, for emerging markets, even bi-monthly return series, which are rebuilt from $d_{3}$, seem to carry information on crashes and events and this seems to imply that emerging markets take up to two months to recover from a crash. Secondly, for mature markets, the ratio in Figures 2(c and d) are meaningless because the ratio scales are very big and this indicates that neither monthly nor bimonthly data, (rebuilt from $d_{2}$ and $d_{3}$ respectively), seem to have information on crises and events implying that mature markets take less than a month to recover from crashes.

To sum up, we would say that the results appear to indicate that mature markets take action more quickly than emerging markets to recover from crashes and also that mature markets exhibit anti-persistent behaviour while emerging markets show persistent behaviour. In other words, the recovery time from crisis for developed markets appears to be shorter than that for developing ones. 


\section{Discussion/Conclusion}

The aims of this work were to study the distribution of the Largest $\left(\lambda_{1}\right)$ and the Second Largest $\left(\lambda_{2}\right)$ eigenvalues of covariance matrices for emerging and mature markets and also to study the distribution of the ratio of $\lambda_{1}$ to $\lambda_{2}$ for the original return series and for those reconstructed from wavelet components $\left(d_{1}, d_{2}\right.$ and $\left.d_{3}\right)$. The summary of our results is as follows:

1. From studying the original return series, we found that differences exist between emerging and mature markets in dealing with crashes (especially unexpected ones). For major markets, the ratio is high at three points representing the $12^{\text {th }}$ anniversary of the October 19 stock market crash, 1999, the 9/11 crash, 2001 and Madrid Bomb, March, 2004 respectively. However, for emerging markets, the ratio is only high at one point, representing the 9/11 crash, 2001 .

2. Using the discrete wavelet transform to study the behaviour of stock markets provides a clearer view on the structure and dynamics of the data sets and gives us a good measurement of the recovery time and direction of movements in these markets. It also indicates that emerging markets take up to two months to recover from crashes while mature ones take less than a month to do so.

3. Both $\lambda_{1}$ and $\lambda_{2}$ are needed to describe the behaviour of emerging markets while $\lambda_{1}$ is adequate alone to describe the behaviour of mature markets.

4. Mature markets move together in the same direction to deal with crises and show little internal variation which suggests that cooperative behaviour applies both within and between such markets. In other words, shareholders in these markets appear to have similar patterns of selling and buying shares. However, emerging markets show more internal variation and thus demonstrate differing views of shareholders in these markets which take different directions in dealing with crashes and unexpected events.

\section{REFERENCES}

1. Bruce, A. and Gao, H. Y. (1996) "Applied Wavelet Analysis with SPlus", New York: Springer-Verlag. 
2. Di Matteo, T., Aste, T. and Dacorogna, M. M. (2003) "Scaling Behaviors in Differently Developed Markets", Physica A, Vol 324,pp 183-188.

3. Di Matteo, T., Aste, T. and Dacorogna, M. M. (2005) "Long-Term Memory of Developed and Emerging Markets: Using the Scaling Analysis to Characterize Their Stage of Development", Journal of Banking \& Finance, Vol 29,pp 827-851.

4. Galluccio, S., Bouchaud, J. P. and Potters, M. (1998) "Rational Decisions, Random Matrices and Spin Glasses", Physica A, Vol 259, pp 449-456.

5. Gonghui, Z., Starck, J. L., Campbell, J. and Murtagh, F. (1999)"The Wavelet Transform for Filtering Financial Data Stream", (available from strule.cs.qub.ac.uk/ ${ }^{\sim}$ gzheng/financial-engineering/finpapermay99.html) [accessed 20 October 2004].

6. Hijmans, H. E. (1993) "Discrete Wavelet and Multiresolution Analysis", Wavelets: An Elementary Treatment of Theory and Application, Koornwinder, T. H. (Editor), (Singapore: World Scientific Publishing Co. Pte.Ltd), pp 49-79.

7. Fuss, R. (2002) "The Financial Characteristics between Emerging and Developed Equity Markets", Policy Modelling,International Conference Brussels, the EcoMod Network, July, 2002.

8. Gopikrishnan, P., Rosenow, B., Plerou, V. and Stanley, E.(2001) "Quantifying and Interpreting Collective behaviour in Financial Markets", Physical Review E, Vol 64, pp 035106(1-4).

9. Keogh, G., Sharifi, S., Ruskin, H. and Crane, M., (2003) "Epochs in Market Sector Index Data - Empirical or Optimistic?", Proceedings of the 2nd Nikkei Econophysics Symposium - Application of Econophysics, Takayasu, H. (Editor), Lecture Notes in Computer Science, Springer, November, 2003, pp 83-89, ISBN 4-431-14028-X

10. Kwapien, J., Drozdz, S. and Speth, J. (2002) "Alternation of Different Scaling Regimes in the Stock Market Fluctuation", (Available from www.fz-juelich.de/ikp/publications/AR2002/CHAP4/409.pdf), [Accessed 11 May 2004].

11. Kwapien, J., Drozdz, S. and Speth, J. (2004) "Time Scale involved in Emergent Market Coherence", Physica A, Vol 337, pp 231-242. 
12. Laloux, L., Cizeau, P. and Potters, M. (2000) "Random Matrix Theory and Financial Correlations",International Journal of Theoretical and Applied Finance (IJTAF), Vol 3 (3), pp 391-397.

13. Laloux, L., Cizeau, P., Bouchaud, J. P. and Potters, M. (1999) "Noise Dressing of Financial Correlation Matrices", Physical Review Letters, Vol 83 (7), pp 1467-1470.

14. Litterman, R. and Winkelmann, K. (1998) "Estimating Covariance Matrices", in Goldman-Sachs Risk Management Series, Krieger. R. A. (Editor), Goldman, Sachs \& Co.

15. Meric, I. and Meric, G. (1997) "Co-movements of European Equity Markets before and after the 1987 crash", Multinational Finance Journal, Vol 1(2), pp 137-152.

16. Pafka, S. and Kondor, I. (2002) "Nosiy Covariance Matrices and Portfolio Optimization", The European Physical Journal B, Vol 27, pp 277280 .

17. Patel, S. and Sarkar, A. (1998) "Stock Market Crises in Developed and Emerging Stock Markets", Federal Reserve Bank of New York, Research Paper, No: 9809.

18. Plerou, V., Gopikrishnan, P. and Rosenow, B., Amaral, L. A. N. and Stanley, H. E. (1999) "Universal and Non-universal Properties of Cross Correlations in Financial Time Series", Physical Review Letters, Vol 83 (7), pp 1471-1474.

19. Plerou, V., Gopikrishnan, P. and Rosenow, B. (2001) "Collective Behaviour of Stock Price Movement: A Random Matrix Theory Approach", Physica A, Vol 299, pp 175-180.

20. Salomons, R. and Grootveld, H. (2002) "The Equity Risk Premium: Emerging versus Developed Markets", (August 7, 2002). University of Groningen SOM Working Paper No. 02E45.

21. Sharifi, S., Crane, M., Shamaie, A. and Ruskin, H. (2004) "Random Matrix Theory for Portfolio Optimization: A Satiability approach",Physica A, Vol 335 (3-4), pp 629-643.

22. Sharkasi, A., Crane, M. and Ruskin, H. J (2004) "Apples and Oranges: the Difference between the Reaction of Emerging and Mature Markets to Crashes", Proceedings of the Third Nikkei Econophysics Research Workshop and Symposium - Practical Fruits of Econophysics, 
Takayasu, H. (Editor), Lecture Notes in Computer Science, Springer (in press).

23. Sornette, D. (2002) "Why stock markets crash: critical events in complex financial systems", Princeton University Press, New Jersey, USA, ISBN: 0-691-09630-9.

24. Wilcox, D. and Gebbie, T. (2004) "On the Analysis of Cross-Correlations in South African Market Data", Physica A: Statistical Mechanics and its Applications, Vol 344 (1-2), Pp 294-298.

25. Wilcox, D. and Gebbie, T. (2004) "An Analysis of Cross-Correlations in South African Market Data", submitted to Physica A, e-print http://arxiv.org/abs/cond-mat/0402389.

26. Wooldridge, P. D., Domanski, D. and Cobau, A. (2003) "Changing Links between Mature and Emerging Financial Markets", BIS Quarterly Review, September 2003, pp 45-54. 


\begin{tabular}{|l|c|c|c|c|c|c|c|}
\hline $\begin{array}{l}\text { W.Crystals } \rightarrow \\
\text { Market } \downarrow\end{array}$ & $d_{1}$ & $d_{2}$ & $d_{3}$ & $d_{4}$ & $d_{5}$ & $d_{6}$ & $s_{6}$ \\
\hline Argentina (Americas) & $\mathbf{0 . 4 1 5}$ & $\mathbf{0 . 2 0 3}$ & $\mathbf{0 . 1 9 2}$ & 0.124 & 0.034 & 0.011 & 0.021 \\
\hline Brazil (Americas) & $\mathbf{0 . 5 2 1}$ & $\mathbf{0 . 1 8 5}$ & $\mathbf{0 . 1 2 4}$ & 0.095 & 0.055 & 0.002 & 0.019 \\
\hline Ireland (Europe) & $\mathbf{0 . 4 4 0}$ & $\mathbf{0 . 2 5 0}$ & $\mathbf{0 . 1 1 5}$ & 0.104 & 0.062 & 0.004 & 0.025 \\
\hline Korea (Asia) & $\mathbf{0 . 5 8 3}$ & $\mathbf{0 . 2 0 7}$ & $\mathbf{0 . 0 7 6}$ & 0.070 & 0.021 & 0.020 & 0.022 \\
\hline Malaysia (Asia) & $\mathbf{0 . 4 9 8}$ & $\mathbf{0 . 2 1 1}$ & $\mathbf{0 . 1 0 7}$ & 0.101 & 0.032 & 0.016 & 0.035 \\
\hline Mexico (Americas) & $\mathbf{0 . 4 5 5}$ & $\mathbf{0 . 2 4 6}$ & $\mathbf{0 . 1 4 4}$ & 0.074 & 0.057 & 0.012 & 0.013 \\
\hline New Zealand (Pacific) & $\mathbf{0 . 5 4 6}$ & $\mathbf{0 . 1 9 7}$ & $\mathbf{0 . 1 2 6}$ & 0.070 & 0.037 & 0.019 & 0.006 \\
\hline Norway (Europe) & $\mathbf{0 . 4 6 9}$ & $\mathbf{0 . 2 4 7}$ & $\mathbf{0 . 1 0 9}$ & 0.076 & 0.059 & 0.022 & 0.018 \\
\hline Portugal (Europe) & $\mathbf{0 . 4 6 1}$ & $\mathbf{0 . 1 9 0}$ & $\mathbf{0 . 1 3 6}$ & 0.084 & 0.079 & 0.020 & 0.030 \\
\hline Russia (Europe) & $\mathbf{0 . 4 3 4}$ & $\mathbf{0 . 2 3 9}$ & $\mathbf{0 . 1 2 6}$ & 0.082 & 0.063 & 0.019 & 0.037 \\
\hline Singapore (Asia) & $\mathbf{0 . 4 9 6}$ & $\mathbf{0 . 2 1 3}$ & $\mathbf{0 . 1 0 6}$ & 0.124 & 0.016 & 0.020 & 0.025 \\
\hline Taiwan (Asia) & $\mathbf{0 . 4 6 5}$ & $\mathbf{0 . 3 0 8}$ & $\mathbf{0 . 1 0 6}$ & 0.051 & 0.043 & 0.009 & 0.019 \\
\hline Turkey (Middle East) & $\mathbf{0 . 4 7 7}$ & $\mathbf{0 . 2 1 3}$ & $\mathbf{0 . 1 4 1}$ & 0.058 & 0.075 & 0.014 & 0.023 \\
\hline
\end{tabular}

Table 1: Emerging Markets: Percentages of energy explained by wavelet components for the original returns series.

\begin{tabular}{|l|c|c|c|c|c|c|c|}
\hline $\begin{array}{l}\text { W.Crystals } \rightarrow \\
\text { Market } \downarrow\end{array}$ & $d_{1}$ & $d_{2}$ & $d_{3}$ & $d_{4}$ & $d_{5}$ & $d_{6}$ & $s_{6}$ \\
\hline Australia (Pacific) & $\mathbf{0 . 4 9 9}$ & $\mathbf{0 . 2 2 6}$ & $\mathbf{0 . 1 6 8}$ & 0.055 & 0.038 & 0.010 & 0.005 \\
\hline Canada (Americas) & $\mathbf{0 . 5 5 2}$ & $\mathbf{0 . 2 0 2}$ & $\mathbf{0 . 1 0 4}$ & 0.050 & 0.054 & 0.028 & 0.011 \\
\hline Denmark (Europe) & $\mathbf{0 . 5 0 5}$ & $\mathbf{0 . 1 5 1}$ & $\mathbf{0 . 2 2 1}$ & 0.044 & 0.026 & 0.033 & 0.020 \\
\hline France (Europe) & $\mathbf{0 . 5 4 6}$ & $\mathbf{0 . 2 3 1}$ & $\mathbf{0 . 1 0 3}$ & 0.055 & 0.025 & 0.019 & 0.022 \\
\hline German (Europe) & $\mathbf{0 . 5 9 4}$ & $\mathbf{0 . 2 1 4}$ & $\mathbf{0 . 1 2 8}$ & 0.031 & 0.023 & 0.007 & 0.004 \\
\hline Hong Kong (Asia) & $\mathbf{0 . 4 8 7}$ & $\mathbf{0 . 2 2 1}$ & $\mathbf{0 . 1 3 8}$ & 0.100 & 0.026 & 0.007 & 0.021 \\
\hline Italy (Europe) & $\mathbf{0 . 5 1 1}$ & $\mathbf{0 . 2 2 0}$ & $\mathbf{0 . 1 4 6}$ & 0.060 & 0.030 & 0.014 & 0.019 \\
\hline Japan (Asia) & $\mathbf{0 . 5 5 7}$ & $\mathbf{0 . 2 1 3}$ & $\mathbf{0 . 1 2 3}$ & 0.059 & 0.020 & 0.010 & 0.019 \\
\hline Netherlands (Europe) & $\mathbf{0 . 3 9 0}$ & $\mathbf{0 . 4 1 8}$ & $\mathbf{0 . 0 6 4}$ & 0.091 & 0.010 & 0.018 & 0.008 \\
\hline Sweden (Europe) & $\mathbf{0 . 5 1 8}$ & $\mathbf{0 . 2 0 1}$ & $\mathbf{0 . 1 3 3}$ & 0.063 & 0.036 & 0.026 & 0.023 \\
\hline Switzerland (Europe) & $\mathbf{0 . 4 5 8}$ & $\mathbf{0 . 2 7 7}$ & $\mathbf{0 . 1 3 3}$ & 0.070 & 0.028 & 0.015 & 0.018 \\
\hline UK (Europe) & $\mathbf{0 . 5 3 2}$ & $\mathbf{0 . 2 4 4}$ & $\mathbf{0 . 1 1 3}$ & 0.054 & 0.032 & 0.011 & 0.013 \\
\hline US (NASDEQ) (Americas) & $\mathbf{0 . 5 3 1}$ & $\mathbf{0 . 2 3 3}$ & $\mathbf{0 . 1 2 1}$ & 0.051 & 0.023 & 0.008 & 0.034 \\
\hline US (S\&P500) (Americas) & $\mathbf{0 . 5 5 0}$ & $\mathbf{0 . 2 2 4}$ & $\mathbf{0 . 1 2 5}$ & 0.051 & 0.025 & 0.009 & 0.017 \\
\hline
\end{tabular}

Table 2: Mature Markets: Percentages of energy explained by wavelet components for the original returns series. 


\begin{tabular}{|c|c|l|l|}
\hline Mark & Window No. & Last week included & Events \\
\hline a1 & 5 & first week of $7 / 1997$ & Asian Crash \\
\hline $\mathbf{a} 2$ & 23 & second week of $11 / 1997$ & Asian Crash \\
\hline a3 & 62 & fourth week of $8 / 1998$ & Global Crash \\
\hline $\mathbf{a} 4$ & 130 & second week of $1 / 2000$ & \\
\hline a5 & 176 & second week of $12 / 2000$ & Effects of DotCom Crash \\
\hline $\mathbf{a} 6$ & 186 & second week of $3 / 2001$ & \\
\hline $\mathbf{a} 7$ & 212 & second week of $9 / 2001$ & September the $11^{\text {th }}$ Crash \\
\hline a8 & 227 & fourth week of $1 / 2002$ & \\
\hline
\end{tabular}

a: Emerging Markets.

\begin{tabular}{|c|c|l|l|}
\hline Mark & Window No. & Last week included & Events \\
\hline b1 & 65 & first week of $9 / 1998$ & Global Crash \\
\hline b2 & 84 & fourth week of $12 / 1998$ & Global Crash \\
\hline b3 & 121 & third week of $10 / 1999$ & Last October in $20^{\text {th }}$ Century \\
\hline b4 & 153 & second week of $6 / 2000$ & DotCom Crash \\
\hline b5 & 220 & second week of $9 / 2001$ & September the $11^{\text {th }}$ Crash \\
\hline b6 & 225 & first week of $11 / 2001$ & Effects of $9 / 11$ Crash \\
\hline b7 & 231 & second week of $12 / 2001$ & Effects of $9 / 11$ Crash \\
\hline b8 & 259 & first week of $5 / 2002$ & The Stock Market Downturn \\
\hline b9 & 322 & first week of $10 / 2003$ & \\
\hline b10 & 331 & first week of $12 / 2003$ & General Threat Level Raised \\
\hline b11 & 345 & third week of $3 / 2004$ & Madrid Bomb \\
\hline
\end{tabular}

b: Mature Markets.

Table 3: Description of the marks in the Figures 3 (a and b) 


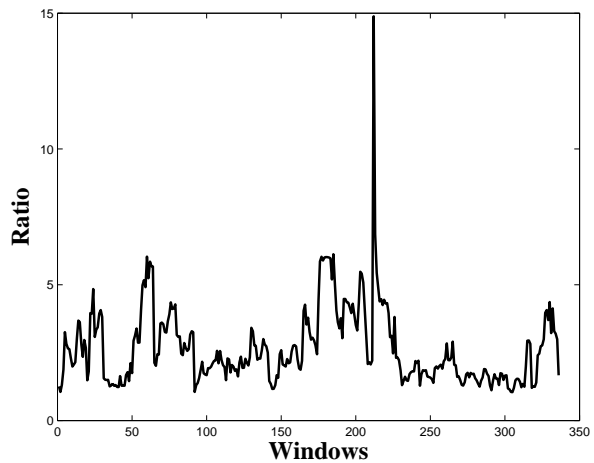

(a) Original Return series.

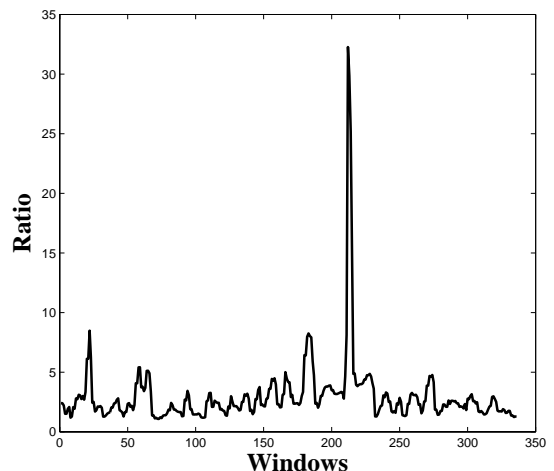

(c) Return series rebuilt from second wavelet crystal $\left(d_{2}\right)$.

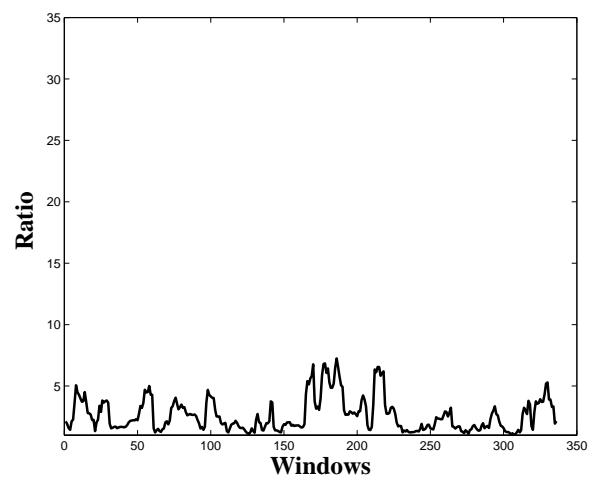

(b) Return series rebuilt from first wavelet crystal $\left(d_{1}\right)$.

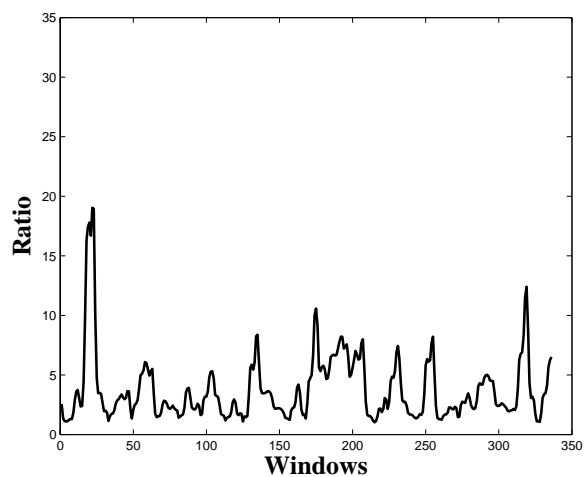

(d) Return series rebuilt from third wavelet crystal $\left(d_{3}\right)$.

Figure 1: Emerging Markets: The changes in ratio of Dominant $\left(\lambda_{1}\right)$ to Subdominant $\left(\lambda_{2}\right)$ eigenvalues. 


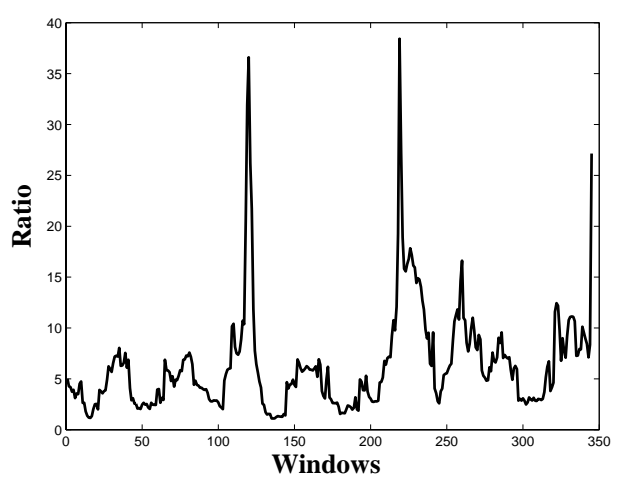

(a) Original Return series.

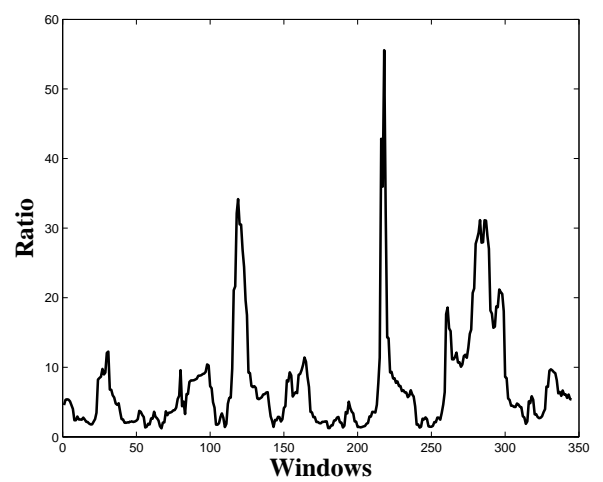

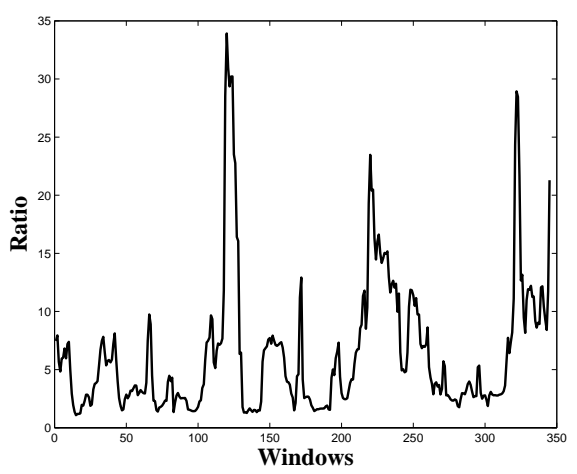

(b) Return series rebuilt from first wavelet crystal $\left(d_{1}\right)$.

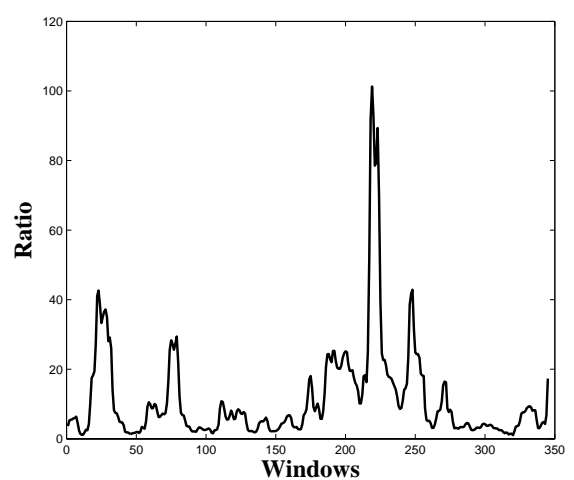

(c) Return series rebuilt from second (d) Return series rebuilt from third wavelet crystal $\left(d_{2}\right)$. wavelet crystal $\left(d_{3}\right)$.

Figure 2: Mature Markets: The changes in ratio of Dominant $\left(\lambda_{1}\right)$ to Subdominant $\left(\lambda_{2}\right)$ eigenvalues. 


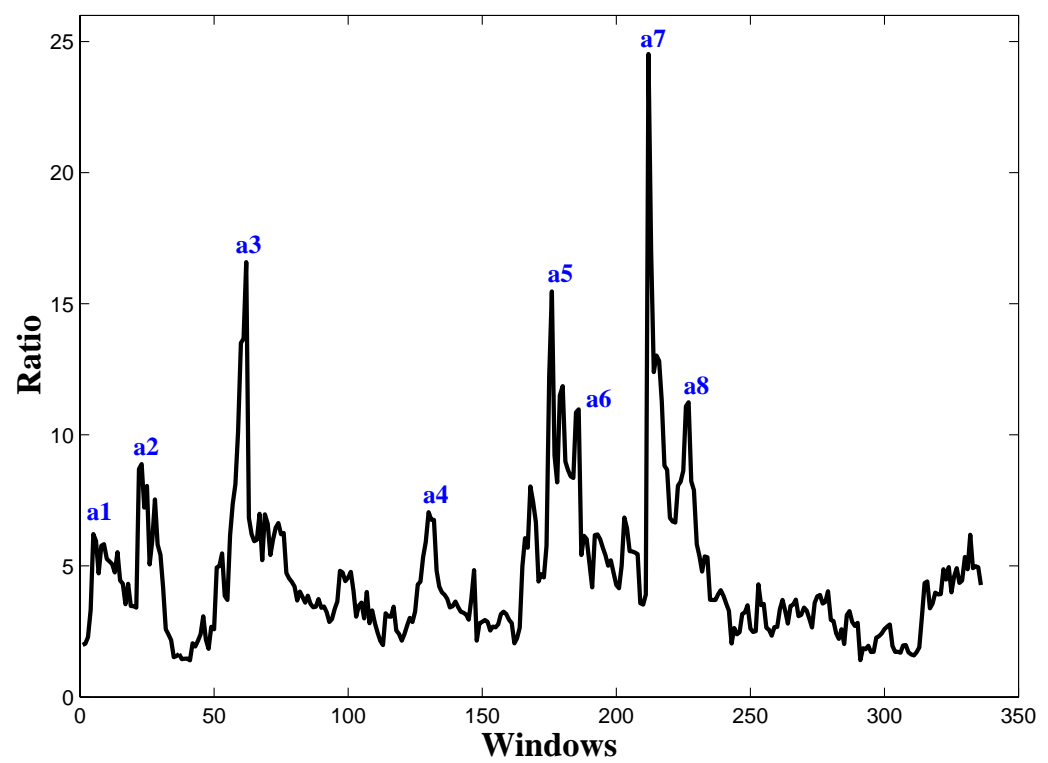

(a) Original Return series for emerging markets.

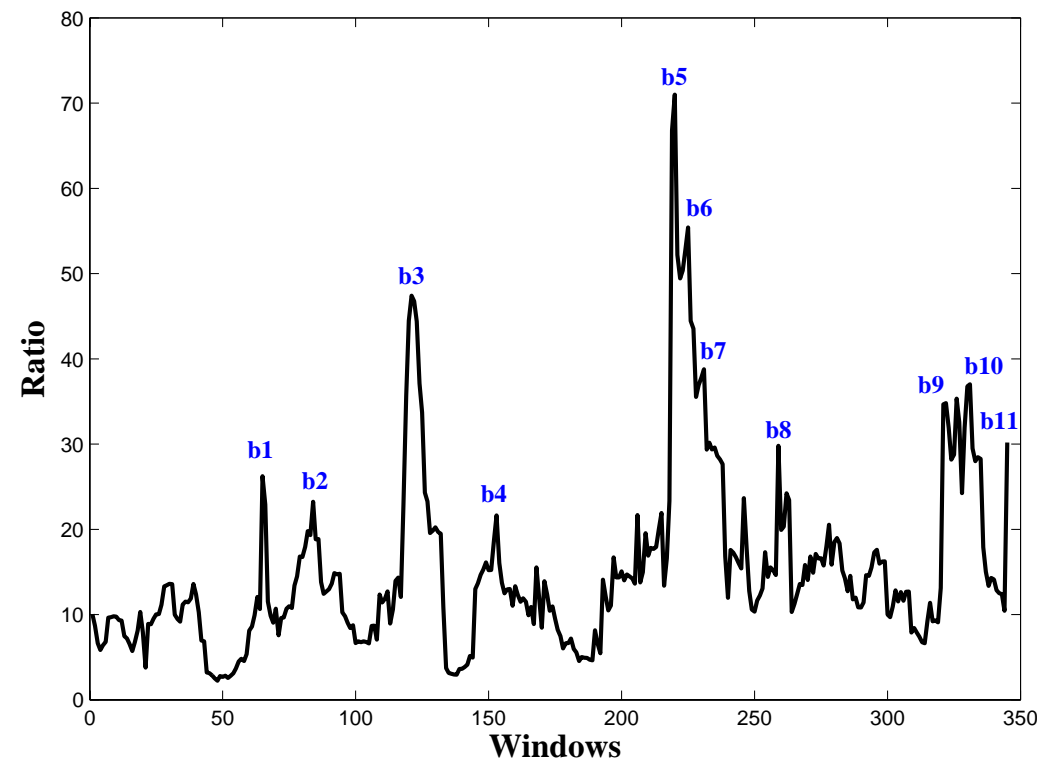

(b) Original Return series for mature markets.

Figure 3: The changes in ratio of the First Largest $\left(\lambda_{1}\right)$ to the Third Largest $\left(\lambda_{3}\right)$ eigenvalues. 


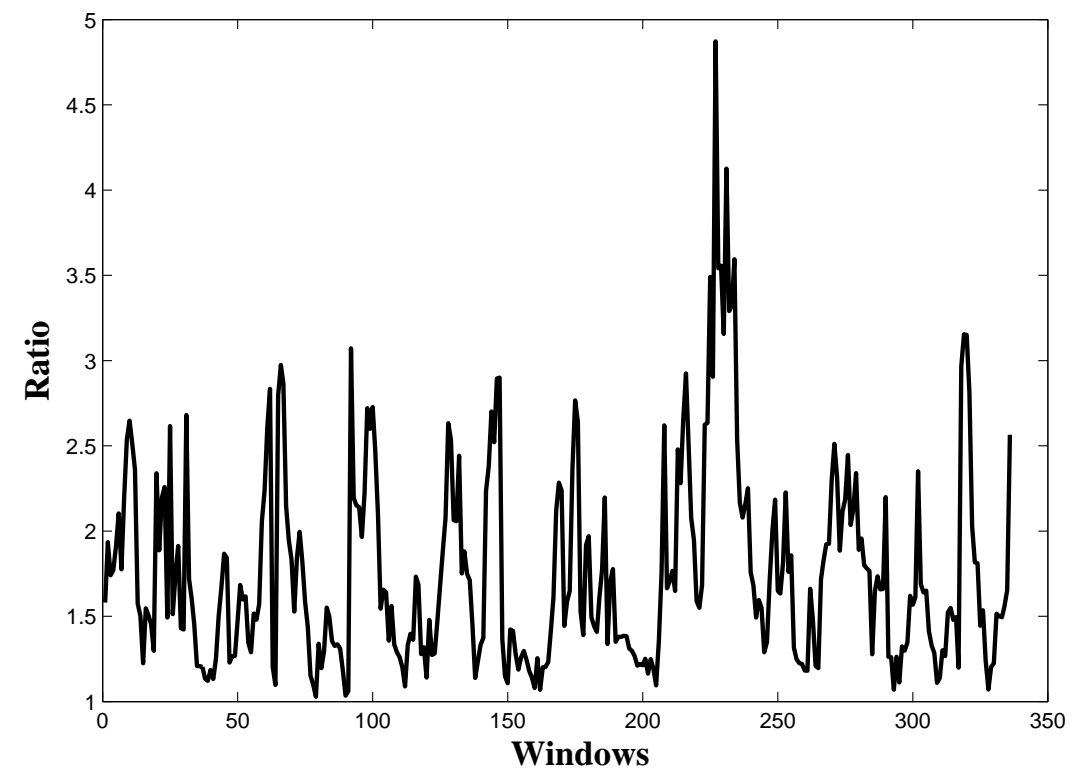

(a) Original Return series for emerging markets.

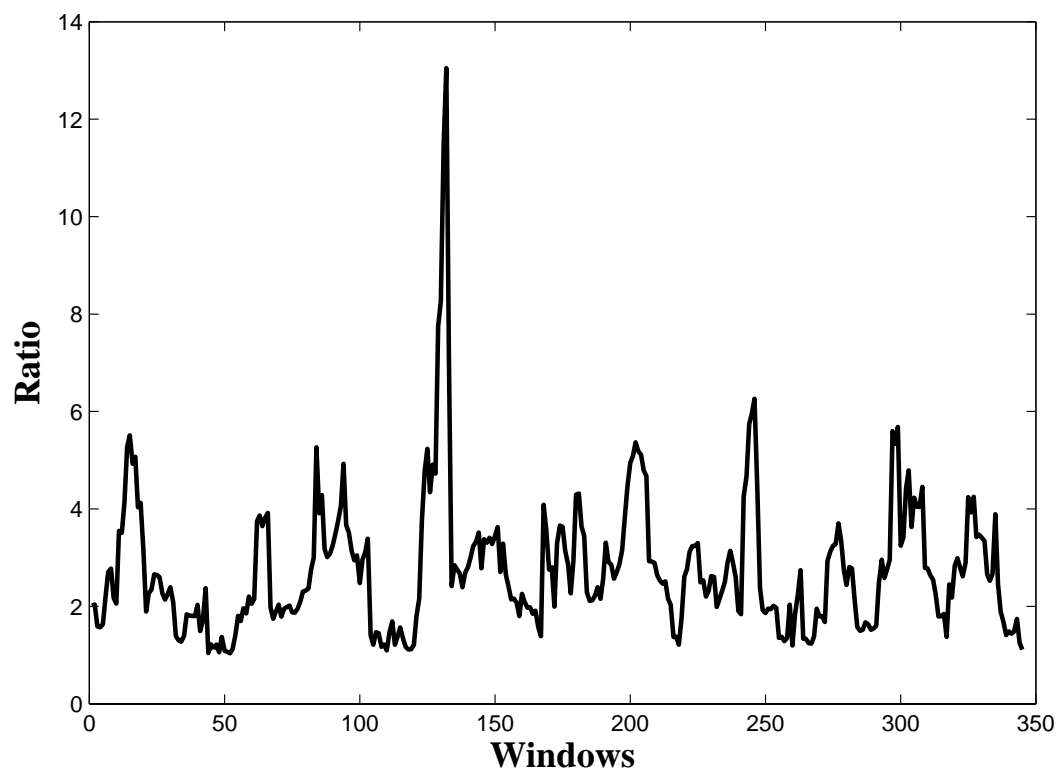

(b) Original Return series for mature markets.

Figure 4: The changes in ratio of the Second Largest $\left(\lambda_{2}\right)$ to the Third Largest $\left(\lambda_{3}\right)$ eigenvalues. 


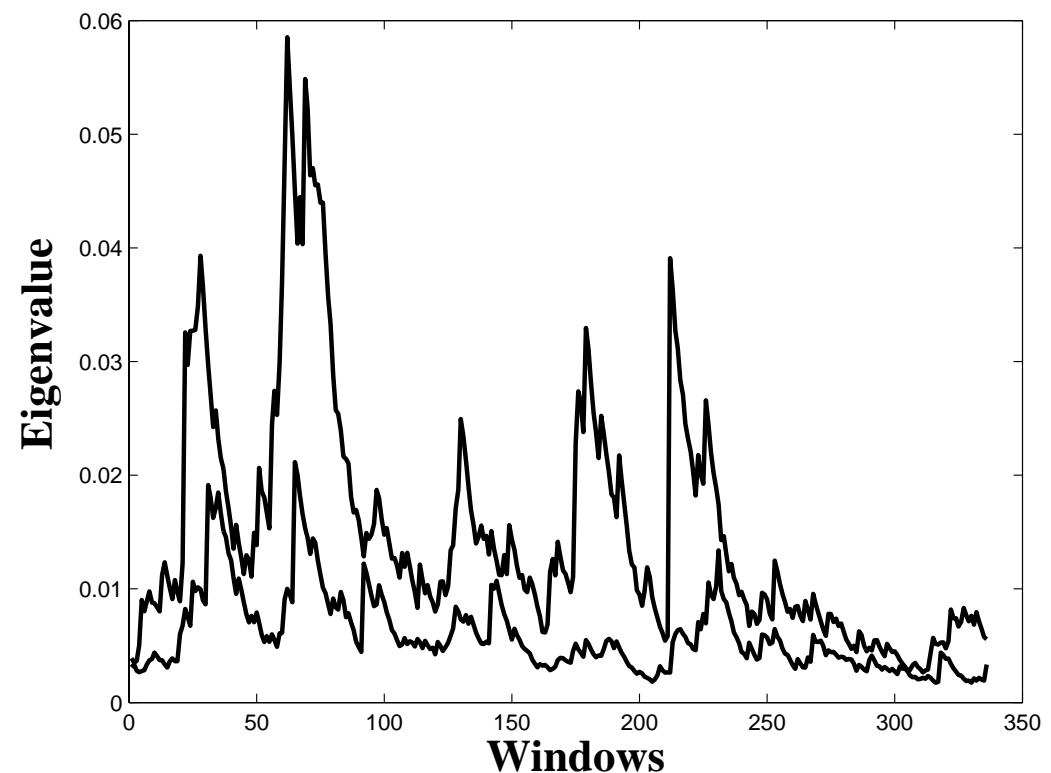

(a) Original Return series for emerging markets.

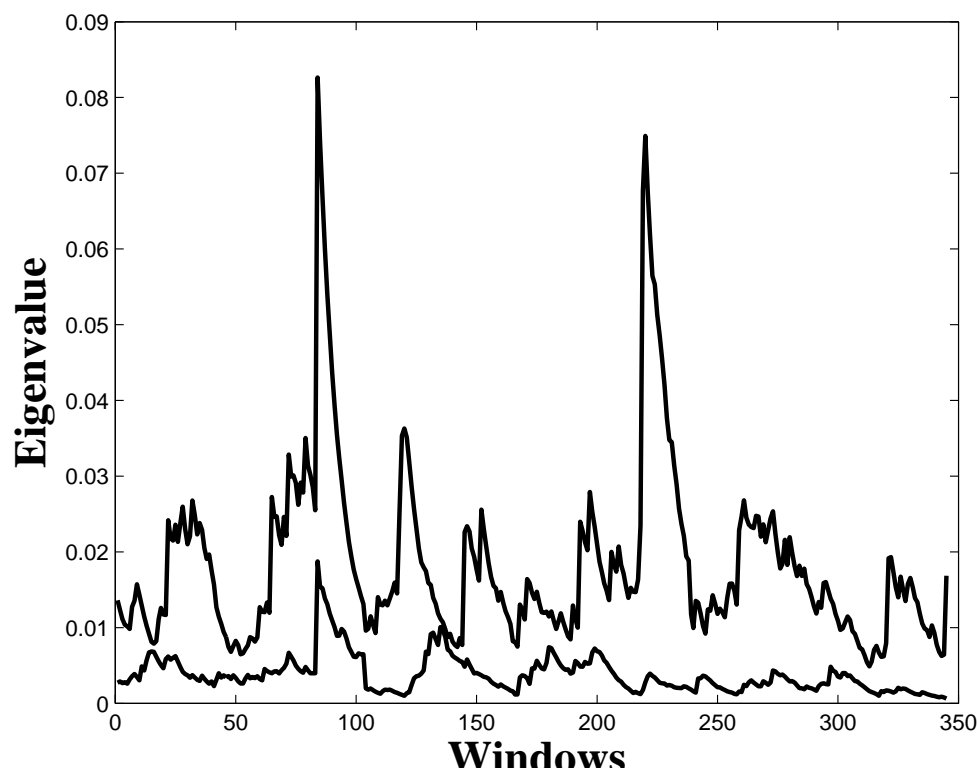

(b) Original Return series for mature markets.

Figure 5: The changes in the Dominant $\left(\lambda_{1}\right)$ (upper line) and the Subdominant $\left(\lambda_{2}\right)$ (lower line)eigenvalues. 